\title{
Chemotherapy during Pregnancy: Cases of Hodgkin's and Non-Hodgkin's Lymphoma, Chronic Myeloid Leukemia, Breast Cancer, Nasopharyngeal Cancer, and Choriocarcinoma
}

\author{
Kemal Beksac ${ }^{a}$ Gokcen Orgul $^{b} \quad$ Ozgur Ozyuncu $^{b} \quad$ Murat Yurdakok $^{c} \quad K^{2}$ adri Altundag ${ }^{d}$ \\ Mehmet S. Beksac ${ }^{\text {b }}$ \\ a Department of General Surgery, Ankara Oncology Hospital, Ankara, Turkey; \\ ${ }^{b}$ Division of Perinatology, Department of Obstetrics and Gynecology, Hacettepe University, Ankara, Turkey; \\ ${ }^{c}$ Division of Neonatology, Department of Pediatrics, Hacettepe University, Ankara, Turkey; \\ ${ }^{\mathrm{d} D i v i s i o n}$ of Medical Oncology, Department of Internal Medicine, Hacettepe University, Ankara, Turkey
}

\section{Keywords}

Pregnancy · Teratology · Hodgkin's lymphoma .

Non-Hodgkin's lymphoma · Chronic myeloid leukemia ·

Nasopharyngeal cancer · Choriocarcinoma

\section{Summary}

Background: The use of chemotherapeutics during pregnancy is a dilemma for both the patient and the clinician. We report here our 11 years' experience with the use of chemotherapy during pregnancy. Patients: 13 patients undergoing chemotherapy during their current pregnancy were evaluated. The medical data of 5 patients with hematologic malignancies (2 Hodgkin's, 2 nonHodgkin's lymphoma, and 1 chronic myeloid leukemia), 6 patients with breast cancer, 1 patients with nasopharyngeal cancer, and 1 patient with choriocarcinoma were retrospectively obtained from the 'perinatal database' of Hacettepe University for the period of January 2002 through March 2016. Results: 4 patients had a medical termination due to teratologic effects. 4 patients had a vaginal delivery, and 5 patients delivered by caesarean section. The 9 newborns who had been exposed to chemotherapeutics were free of congenital anomalies. However, 1 of them died in the early neonatal period due to multi-organ failure (nasopharyngeal cancer). During the 2-year follow-up, we encountered 1 maternal mortality, which was due to multi-organ failure in a non-Hodgkin's lymphoma patient. Conclusion: Physicians must pay attention to potential teratologic problems, and should carefully balance maternal and fetal risks. Se- lected chemotherapeutic agents can be given in the 2 nd and 3rd trimester without any major teratogenic risk. All 9 newborns in our study were free of anomalies, although 1 of them died in the neonatal period.

(c) 2017 S. Karger GmbH, Freiburg

\section{Introduction}

Cancer is the second most common cause of death among women of child-bearing age worldwide. With the increasing participation of women in the workforce, childbearing is postponed, contributing to the rise in pregnancies associated with cancer [1]. Chemotherapy exposure during pregnancy is a great concern for both the mother and the physician. Due to their relatively low molecular weight, chemotherapeutic agents can cross the placenta and adversely affect the fetus [2-4]. Teratologic and other adverse fetal effects are dependent on dosage and the gestational age [5].

Exposure during the 1st trimester, in which organogenesis takes place, may result in teratogenesis and spontaneous abortions $[3,6]$. Some organs and systems, including the eyes and genitalia, the hematopoietic system, and the central nervous system, remain vulnerable throughout pregnancy [7]. Subsequent trimesters are relatively safe with an increased risk of stillbirth, intrauterine growth restriction, prematurity, and low birth weight. A limited number of drugs are proven human teratogens and are contraindicated during pregnancy. Older-generation alkylators such as procarbazine, busulfan, chlorambucil, and nitrogen mustard have high terato-

\section{KARGER}

(c) 2017 S. Karger GmbH, Freiburg 
Table 1. Patient characteristics

\begin{tabular}{|c|c|c|c|c|c|c|c|c|c|}
\hline $\begin{array}{l}\text { Case, } \\
\mathrm{n}\end{array}$ & Type of cancer & $\begin{array}{l}\text { Age, } \\
\text { years }\end{array}$ & $\begin{array}{l}\text { Gestational week at } \\
\text { cancer diagnosis/ } \\
\text { chemotheraphy exposure }\end{array}$ & $\begin{array}{l}\text { Gestational } \\
\text { week of } \\
\text { delivery }\end{array}$ & $\begin{array}{l}\text { Type of } \\
\text { delivery }\end{array}$ & $\begin{array}{l}\text { Birth } \\
\text { weight, } g\end{array}$ & $\begin{array}{l}\text { Newborn's } \\
\text { sex }\end{array}$ & Chemotherapy & $\begin{array}{l}\text { Perinatal } \\
\text { outcome }\end{array}$ \\
\hline 1 & Hodgkin's lymphoma & 30 & before pregnancy/18 & 40 & NVD & 3,410 & male & ABVD & NVD \\
\hline 2 & Hodgkin's lymphoma & 34 & before pregnancy/13 & 15 & TA & NA & female & ABVD & terminated \\
\hline 3 & NHL & 30 & before pregnancy/12 & 16 & TA & NA & male & R-CHOP & terminated \\
\hline 4 & NHL & 24 & $24 / 26$ & 28 & NVD & 1,750 & female & methotrexate & NVD \\
\hline 5 & CML & 26 & $16 / 21$ & 35 & $\mathrm{C} / \mathrm{S}$ & 2,750 & female & hydroxyurea & NVD \\
\hline 6 & breast cancer & 36 & $29 / 30$ & 39 & NVD & 3,200 & female & CAF & NVD \\
\hline 7 & breast cancer & 43 & $24 / 28$ & 37 & NVD & 2,870 & female & CAF & NVD \\
\hline 8 & breast cancer & 39 & before pregnancy/21 & 21 & TA & NA & NA & CAF & terminated \\
\hline 9 & breast cancer & 23 & 06.Haz & 8 & TA & NA & NA & CAF & terminated \\
\hline 10 & breast cancer & 33 & before pregnancy/1 & 38 & $\mathrm{C} / \mathrm{S}$ & 2,560 & female & tamoxifen & NVD \\
\hline 11 & breast cancer & 33 & $17 / 25$ & 34 & $\mathrm{C} / \mathrm{S}$ & 2,650 & female & CAF & NVD \\
\hline 13 & choriocarcinoma & 28 & $27 / 30$ & 35 & $\mathrm{C} / \mathrm{S}$ & 2,890 & female & EMA/CO & NVD \\
\hline
\end{tabular}

$\mathrm{NHL}=$ Non-Hodgkin's lymphoma; CML = chronic myeloid leukemia; NVD = normal vaginal delivery; $\mathrm{C} / \mathrm{S}=\mathrm{caesarian}$ section; $\mathrm{NA}=$ not applicable; TA = terminated; $\mathrm{ABVD}=$ adriamycin, bleomycin, vinblastine, dacarbazine; $\mathrm{CAF}=$ cyclophosfamide, adryamicin, 5-fluorouracil; EMA/CO = etoposide, methotrexate, actinomycin D, cyclophosphamide, vincristine; R-CHOP = rituximab- cyclophosfamide, adryamicin, vincristine, prednisone.

genicity compared to anthracyclines and vinca alkaloids (vinblastine, vincristine) $[4,8]$. Higher doses with multi-drug regimens are reported to be more fetotoxic.

We have evaluated our experience with women undergoing chemotherapy in the course of their pregnancies in regard to demographics and fetal and maternal outcomes.

\section{Patients and Methods}

This retrospective study was carried out at the Division of Perinatology, Department of Obstetrics \& Gynecology, Hacettepe University, Ankara. The period between January 2002 and March 2016 was screened, and 13 patients who received chemotherapy during their pregnancy were enrolled into the study. Patients' data were collected from the 'perinatology database' of Hacettepe University, which comprises information recorded during the antenatal visits.

The study group consisted of 5 patients with hematologic malignancies (2 Hodgkin's lymphomas (HL), 2 non-Hodgkin's lymphomas (NHL), and 1 chronic myeloid leukemia (CML)), 6 breast cancer patients, 1 patient with nasopharyngeal cancer, and 1 patient with choriocarcinoma.

The $2 \mathrm{HL}$ patients received the ABVD (adriamycin, bleomycin, vinblastine, dacarbazine) regimen. 1 of the 2 NHL patients received R-CHOP (rituximabcyclophosphamide, adriamycin, vincristine, prednisone) chemotherapy, while the other had a primary nervous system lymphoma and was treated with intrathecal methotrexate. The CML patient required a mild dose of hydroxyurea to control leukocytosis.

The breast cancer patients were mostly locally advanced cases, requiring neoadjuvant CAF (cyclophosphamide, adriamycin, 5-fluorouracil) chemotherapy. The nasopharyngeal carcinoma patient was successfully treated with cisplatin and concurrent radiotherapy. The choriocarcinoma patient was diagnosed during pregnancy and received EMA/CO (etoposide, methotrexate, actinomycin D, cyclophosphamide, vincristine) chemotherapy during her 3rd trimester.

Clinical characteristics including demographics, pregnancy outcomes, neonatal outcomes, type of cancer, treatment modalities performed (chemotherapy, radiotherapy, surgery), and obstetrics/perinatal complications were evaluated. Therapeutic abortion is defined as the termination of pregnancy to preserve maternal health before the 22nd completed week of pregnancy. Preterm birth is defined as delivery before the 37 th week of gestation.
Statistical analysis was performed using SPSS (IBM Corp., Armonk, NY, USA).

The study was approved by the institutional ethics committee (GO/16/217). All patients gave written informed consent.

\section{Results}

\section{General Characteristics}

The characteristics of each patient are outlined in detail in table 1 . The mean maternal age was $31.7 \pm 5.75$ years (range $23-43$ years). 5 patients were primiparous and 8 were multiparous. 6 (46.1\%) patients were diagnosed with cancer before their pregnancy and 7 (53.9\%) during their pregnancy. 1 patient was diagnosed during the 1 st trimester, 4 patients during the 2 nd trimester, and 1 patient during the 3 rd trimester. The mean gestational age at diagnosis in the patients who successfully delivered was $35.6 \pm 3.5$ weeks (range 28-40 weeks). 5 patients had hematologic malignancies, 6 patients had breast cancer, 1 patient had choriocarcinoma, and 1 patient had nasopharyngeal cancer. The mean gestational age at chemotherapeutic exposure was $20.5 \pm 7.8$ weeks (range 6-30 weeks).

A total of 4 patients had a medical termination due to teratologic effects (fetal toxicity). 4 patients had vaginal deliveries, and 5 patients delivered by caesarean section. There were 4 (44.4\%) preterm deliveries, which is a much higher percentage than seen among the general population (10\%), with an early and late preterm ratio of $1 / 3$. The mean birth weight was 2,684.4 $\pm 515.8 \mathrm{~g}$ (range 1,750-3,410 g).

The 9 newborns who had been exposed to chemotherapeutics were free of congenital anomalies. However, 1 of them died in the early neonatal period due to multi-organ failure. During the 2-year follow-up, we encountered 1 maternal mortality, which was due to multi-organ failure in an NHL patient. 


\section{Cases}

Among the patients with hematologic malignancies, 2 patients had HL, 2 had NHL, and 1 had CML. Both HL patients were diagnosed with a nodular sclerosing subtype before their pregnancies, and their pregnancies were unplanned. The first patient received the ABVD protocol at the 18th gestational week as well as involved-site radiotherapy at a dose of 30 Gy shortly after delivery. The other HL patient requested a termination of her pregnancy and received $A B V D$ chemotherapy at the 13th gestational week.

Of the NHL patients, 1 was diagnosed with diffuse large B-cell lymphoma before her pregnancy. R-CHOP and involved-field radiotherapy were planned. However, the patient did not accept the risk of receiving radiotherapy during pregnancy or of postponing treatment until after the pregnancy. Her pregnancy was thus terminated 4 weeks after chemotherapy exposure. The other NHL patient was diagnosed with primary central nervous system lymphoma at the 24th gestational week and received intrathecal methotrexate 2 weeks later. The patient delivered a healthy baby at 28 weeks gestation but unfortunately died 2 years later due to disease progression.

The CML patient was diagnosed at 16 weeks gestation. This patient required a mild dose of hydroxyurea to control leukocytosis. She delivered a healthy baby at 35 weeks. Conventional treatment started after delivery.

Of the 6 breast cancer patients, 2 were diagnosed during the 3rd trimester. Both presented with large masses in their breasts, and Tru-Cut biopsy revealed breast cancer. Since both patients had locally advanced disease, they received CAF chemotherapy during the 3rd trimesters and modified radical mastectomy (MRM) after delivery. Neither of them received radiotherapy postnatally. The 3 rd patient was diagnosed during a routine screening mammography just before conceiving by chance, and underwent MRM during the 1st trimester. Her pregnancy was terminated at approximately 22 weeks gestation shortly after chemotherapy treatment by request of the family and due to teratologic reasons. The 4 th patient was a 23 -year-old woman who presented with a palpable mass in her breast (in early pregnancy). Ultrasonography and magnetic resonance imaging (MRI) revealed a multifocal breast cancer. She received neoadjuvant $\mathrm{CAF}$ chemotherapy, and again the pregnancy was terminated 2 weeks later. Afterwards, the patient underwent MRM and radiotherapy. Of the remaining 2 patients, 1 was a 33 -year-old women diagnosed with breast cancer during the 2nd trimester. She had locally advanced disease and received neoadjuvant CAF chemotherapy during her pregnancy. After delivery, she underwent MRM and radiotherapy. The last patient had been first diagnosed with breast cancer 3 years earlier and was undergoing treatment with radiotherapy and tamoxifen. Her pregnancy was discovered quite late at 24 weeks gestation. Further radiotherapy was postponed, and she received tamoxifen for the rest of her pregnancy. She successfully delivered a healthy baby at the 38th gestational week. Radiotherapy recommenced after delivery.

The nasopharyngeal cancer patient was a 33-year-old woman who was diagnosed just prior to her first pregnancy. Her treatment was postponed until the 21st gestational week when she received cisplatin and concurrent radiotherapy. She delivered at 35 weeks by cesarean section. However, the baby died in the early neonatal period due to a nosocomial infection and subsequent multi-organ failure. This was probably due to immunosuppression secondary to radiotherapy.

The choriocarcinoma patient was a multiparous 28-year-old woman who was diagnosed during the 3 rd trimester. The patient received EMA/CO chemotherapy at the 30th gestational week and delivered a healthy baby at 35 weeks. She was screened for metastasis after pregnancy and none was detected.

\section{Discussion}

Cancer treatment during pregnancy is an issue of increasing importance since more women become pregnant in their late 30s. Here, we are reporting the cases of 11 patients who received chemotherapy during their pregnancy.

\section{Hematologic Malignancies}

A total of 5 patients had hematologic malignancies and received chemotherapy accordingly. The incidence of hematologic malignancies during pregnancy is $0.02 \%$ [4]. Hematologic malignancies often necessitate prompt therapy, thus creating a situation where the effects of treatment on the fetus are to be weighed against the welfare of the mother [9].

Of the patients with hematologic malignancies, 3 were diagnosed just prior to an unexpected pregnancy and had to undergo chemotherapy at the end of the 1st trimester. 2 patients who received chemotherapy at week 12 and 13 gestation, had a therapeutic abortion 2 weeks later. The 3 rd patient, who received chemotherapy at 18 weeks, completed her pregnancy successfully.

$\mathrm{HL}$ is reported to be more frequent than NHL in pregnant patients. In fact, about $3 \%$ of HL patients present with concurrent pregnancy. These patients are diagnosed with same-stage disease as non-pregnant patients [10]. NHL is more problematic in this regard as patients may be diagnosed at later stages because pregnancy may masquerade symptoms [11]. An analysis of 121 pregnancy-associated NHL cases revealed that $75 \%$ of patients had stage IV disease with $49 \%$ having reproductive organ involvement. Fetal involvement was rare [12]. In this study, we had 2 HL and 2 NHL cases, and our results were similar to those of previous reports. Both HL patients were diagnosed with a nodular sclerosing subtype before their pregnancies, and their pregnancies were unplanned. The 1st patient received the ABVD protocol at 18 weeks as well as involved-site radiotherapy at a dose of 30 Gy shortly after delivery. The other HL patient requested a termination and received ABVD chemotherapy at 13 weeks. 1 of the NHL patients was diagnosed with diffuse large B-cell lymphoma before her pregnancy. R-CHOP and involved-field radiotherapy were planned for this patient. However, she did not accept the risk of receiving radiotherapy during pregnancy or of postponing treatment until after her pregnancy. The pregnancy was thus terminated 4 weeks after chemotherapy exposure. The other NHL patient was diagnosed 
with primary central nervous system lymphoma at 24 weeks gestation and received intrathecal methotrexate 2 weeks later. She delivered a healthy baby at 28 weeks but unfortunately died 2 years later due to disease progression.

Radiotherapy is usually used in combination with chemotherapy for both HL and NHL. Using radiotherapy during pregnancy is a difficult choice. Fetal exposure may cause growth retardation, malformations, and death. There is even the risk of long-term adverse effects, such as mental retardation, sterility, and childhood cancer. Radiotherapy is generally contraindicated during the 1st trimester [13] and continues to be dangerous thereafter. Exposure to doses $\geq 100 \mathrm{mSv}$ (especially $\geq 500 \mathrm{mSv}$ ) is associated with an increased risk of malformation and mental retardation. After 25 weeks, $\leq 1 \mathrm{~Sv}(1,000 \mathrm{mSv})$ is considered relatively safe, depending on the radiation site [14]. 2 of the $4 \mathrm{HL}$ and NHL patients received radiotherapy. However, 1 patient underwent radiotherapy after her pregnancy and the other after an induced abortion. There are other reports of successful systemic therapy for HL and NHL after the 1st trimester $[15,16]$. This is explained by the fact that chemotherapy is administered after organogenesis and fetal protection is provided by the placental barrier [16].

CML occurs in approximately 1 of 100,000 pregnancies [4]. Treatment with thyrosine kinase inhibitors (TKI) is the standard of care in CML patients. However, TKIs are contraindicated during pregnancy. Only few investigators have suggested TKI use despite the known teratogenic effects $[17,18]$. Other therapeutic approaches such as leukapheresis and interferon (IFN)- $\alpha$ can be considered during pregnancy. Leukapheresis can be effective in decreasing cell count; however, it must be performed frequently, 2-3 times per week. Blood counts tend to drop during the 3rd trimester, allowing less frequent leukapheresis $[19,20]$. IFN- $\alpha$ is a high-molecular-weight molecule and considered safe for use during pregnancy [21]. However, it should be noted that when IFN- $\alpha$ was the first-line therapy for CML, it did not always induce good hematologic control and required supplementation with hydroxyurea, which is a known teratogenic agent. Hydroxyurea is known to cause craniofacial and spinal defects in many species, but seems less damaging in humans [22]. The patient in our series had to receive hydroxyurea to control leukocytosis. IFN- $a$ was initially planned for the patient, but unfortunately she could not tolerate it. Leukapheresis was unavailable at the time, so the patient had to be treated with a mild dose of hydoxyurea at the end of the 2 nd trimester.

\section{Breast Cancer}

Breast cancer is one of the most common cancers seen in pregnant women, and $0.2-3.8 \%$ of breast cancers occur during pregnancy [23]. Pregnancy-associated breast cancer mostly presents as advanced-stage disease, and there can be substantial diagnostic delay [24]. Treatment should be tailored to the clinical presentation and the trimester of pregnancy. Surgery can be performed during all trimesters [25]. There is debate about the adjuvant therapy. Much of the literature on the pharmacologic treatment of breast cancer during pregnancy is anecdotal. Some authors support treatment with CAF chemotherapy during the 2nd and 3rd trimes- ter and delaying radiotherapy until after delivery [26]. However, there is also a consensus report that radiotherapy can be considered during the 1 st and 2 nd trimester but should be postponed during the 3 rd trimester [25]. 2 of the 6 breast cancer patients in our series were diagnosed during the 3rd trimester. Both received CAF chemotherapy without radiotherapy (neither during nor after their pregnancy) and successfully completed their pregnancy without neonatal morbidity and mortality. The 3rd patient was diagnosed during a routine screening mammography just before getting pregnant by chance, and received MRM during the 1st trimester. Her pregnancy was terminated at 22 weeks gestation, just after chemotherapy, by request of the family and for teratologic reasons. The 4th patient was a 23-year-old patient who presented with a palpable mass in her breast (early in pregnancy). Ultrasonography and MRI revealed a multifocal breast cancer. She received neoadjuvant CAF chemotherapy, and again the pregnancy was terminated 2 weeks later. She received MRM and radiotherapy after the termination. The 5th patient similarly presented with a palpable mass. Cancer was diagnosed through ultrasound-guided biopsy, and she received neoadjuvant CAF chemotherapy. The last patient was different in that she presented with cranial metastasis after having undergone surgery for breast cancer 3 years earlier. She was currently undergoing radiotherapy and tamoxifen treatment. Amenorrhea due to the medication meant that her pregnancy was detected late. Despite radiotherapy during the 1st and 2nd trimester and tamoxifen treatment, this patient delivered a healthy baby.

CAF is the preferred chemotherapy during pregnancy. In a prospectively designed study by the MD Anderson Cancer Center, 24 patients were managed with the CAF protocol comprising doxorubicin $50 \mathrm{mg} / \mathrm{m}^{2}$ continuous infusion over a period of $72 \mathrm{~h}$, cyclophosphamide $500 \mathrm{mg} / \mathrm{m}^{2}$ on day 1 , and bolus fluorouracil $500 \mathrm{mg} /$ $\mathrm{m}^{2}$ on days 1 and 4.2 patients had recurrent disease, and 1 patient received chemotherapy during the 1st trimester. No antepartum complications attributable to systemic therapy were noted [27]. Another study, which consisted of the 18-year experience of 5 centers, included 28 patients; 16 patients received CAF and 12 received CMF (cyclophosphamide, methotrexate, fluorouracil). 1 patient received chemotherapy during the 1st trimester of pregnancy which ended in spontaneous abortion. There were no serious adverse consequences for the mothers or the neonates in the other cases [23].

Generally speaking, breast cancer patients in our series were locally advanced cases requiring neoadjuvant chemotherapy and MRM mostly after delivery or in conjunction with a termination.

\section{Nasopharyngeal Carcinoma}

Pregnancy-associated nasopharyngeal carcinoma is associated with poor survival. Recent advances in radiation technology, imaging techniques, and chemotherapy have improved survival, and there are reports of successful pregnancies $[28,29]$. The patient in our series was diagnosed towards the end of the 2nd trimester and received chemotherapy along with concomitant radiotherapy. While there was a successful delivery at 35 weeks gestation, the newborn died in the early neonatal period, probably due to the effects of radiotherapy. 


\section{Choriocarcinoma}

Choriocarcinoma is frequently preceded by a complete mole, an ectopic pregnancy, a non-molar intrauterine abortion, or, uncommonly, by a partial mole. Choriocarcinoma coexisting with or after a 'normal' pregnancy has an incidence of 1 per 160,000 pregnancies [30]. It is associated with poor prognosis and rapid spreading of fatal disease; however, it is curable if diagnosed early and treated accordingly $[31,32]$. The choriocarcinoma patient in our series was diagnosed during the $3 \mathrm{rd}$ trimester at an early stage. She received chemotherapy without delay, and delivered a healthy baby at 35 weeks gestation.

\section{Conclusion}

Selected chemotherapeutic agents can be given in the 2nd and 3 rd trimester without major teratogenic risk. All 9 newborns in our study were free of anomalies, although 1 of them died in the neonatal period. Nonetheless, clinicians should take care to balance the maternal and fetal risks. Maternal wellbeing should take priority.

\section{Disclosure Statement}

The authors declare no conflict of interest. This study is not financially funded. The data was partially presented as a poster at the 11th World Congress of Perinatal Medicine. Both newly and previously presented patients are described in more detail in this manuscript.

\section{References}

1 Turgal M, Beksac K, Basaran D, Yazicioglu A, Ozyuncu O, Aran O, Beksac M: Pregnancy after cancer treatment and pregnancy associated cancer: a single center experience with 96 cases. Gynecology Obstetrics \& Reproductive Medicine 2015;21:93-96.

2 Koren G, Carey N, Gagnon R, Maxwell C, Nulman I, Senikas V: Cancer chemotherapy and pregnancy. J Obstet Gynaecol Can 2013;35:263-280.

3 Amant F, Han SN, Gziri MM, Vandenbroucke T, Verheecke M, van Calsteren K: Management of cancer in pregnancy. Best Pract Res Clin Obstet Gynaecol 2015; 29:741-753

4 Lishner M, Avivi I, Apperley JF, Evens AM, Fumagalli M, Nulman I, Oduncu FS, Peccatori FA, Robinson S, van Calsteren K, Vandenbroucke T, van den Heuvel F, Amant F: Hematologic malignancies in pregnancy: management guidelines from an International consensus meeting. J Clin Oncol 2016;34:501-508.

5 Framarino-Dei-Malatesta M, Perrone G, Giancotti A, Ventriglia F, Derme M, Iannini I, Tibaldi V, Galoppi P, Sammartino P, Cascialli G, Brunelli R: Epirubicin: a new entry in the list of fetal cardiotoxic drugs? Intrauterine death of one fetus in a twin pregnancy. Case report and review of literature. BMC Cancer 2015;15:951.

6 Albright CM, Wenstrom KD: Malignancies in pregnancy. Best Pract Res Clin Obstet Gynaecol 2016;33: 2-18.

7 Cardonick E, Iacobucci A: Use of chemotherapy during human pregnancy. Lancet Oncol 2004;5:283-291.

8 Azim HA Jr, Pavlidis N, Peccatori FA: Treatment of the pregnant mother with cancer: a systematic review on the use of cytotoxic, endocrine, targeted agents and immunotherapy during pregnancy. Part II: Hematological tumors. Cancer Treat Rev 2010;36:110-121.

9 Lavi N, Horowitz NA, Brenner B: An update on the management of hematologic malignancies in pregnancy. Womens Health 2014;10:255-266.

10 Bachanova V, Connors JM: Hodgkin lymphoma in pregnancy. Curr Hematol Malig Rep 2013;8:211-217.

11 Gui L, Shi Y, He X, Zhou L, Dong M, Zhou S, Yuan P, Liu P, Yang J, Zhang C, Qin Y, Yang S: (Clinicopathological features and outcomes: analysis of 21 cases of lymphoma in pregnancy). Zhonghua Yi Xue Za Zhi 2015;95:425-429.

12 Rizack T, Mega A, Legare R, Castillo J: Management of hematological malignancies during pregnancy. Am J Hematol 2009;84:830-841.
13 Streffer C, Shore R, Konermann G, Meadows A, Uma Devi P, Preston Withers J, Holm LE, Stather J, Mabuchi KHR: Biological effects after prenatal irradiation (embryo and fetus). A report of the International Commission on Radiological Protection. Ann ICRP 2003;33:5-206.

14 Otake M, Schull WJ: Radiation-related brain damage and growth retardation among the prenatally exposed atomic bomb survivors. Int J Radiat Biol 1998;74:159171.

15 Pinnix CC, Osborne EM, Chihara D, Lai P, Zhou S, Ramirez MM, Oki Y, Hagemeister FB, Rodriguez AM, Samaniego F, Fowler N, Romaguera JE, Turturro F, Fayad L, Westin JR, Nastoupil L, Neelapu SS, Cheah CY, Dabaja BS, Milgrom SA, Smith GL, Horace P, Milbourne A, Wogan CF, Ballas L, Fanale MA: Maternal and fetal outcomes after therapy for Hodgkin or nonHodgkin lymphoma diagnosed during pregnancy. JAMA Oncol 2016;2:1065-1069.

16 Van Calsteren K: Chemotherapy during pregnancy: pharmacokinetics and impact on foetal neurological development. Facts Views Vis Obgyn 2010;2:278-286.

17 Palani R, Milojkovic D, Apperley JF: Managing pregnancy in chronic myeloid leukaemia. Ann Hematol 2015;94(suppl 2):S167-176.

18 Ali R, Ozkalemkas F, Ozcelik T, Ozkocaman V, Ozan U, Kimya Y, Koksal N, Gulten T, Yakut T, Tunali A: Pregnancy under treatment of imatinib and successful labor in a patient with chronic myelogenous leukemia (CML). Outcome of discontinuation of imatinib therapy after achieving a molecular remission. Leuk Res 2005;29:971-973.

19 Yellu M, Pinkard S, Ghose A, Medlin S: CML in pregnancy: a case report using leukapheresis and literature review. Transfus Apher Sci 2015;53:289-292.

20 Ali R, Ozkalemkas F, Ozkocaman V, Ozcelik T, Ozan U, Kimya Y, Tunali A: Successful pregnancy and delivery in a patient with chronic myelogenous leukemia (CML), and management of CML with leukapheresis during pregnancy: a case report and review of the literature. Jpn J Clin Oncol 2004;34:215-217.

21 Yazdani Brojeni P, Matok I, Garcia Bournissen F, Koren G: A systematic review of the fetal safety of interferon alpha. Reprod Toxicol 2012;33:265-268.
22 Thauvin-Robinet C, Maingueneau C, Robert E, Elefant E, Guy H, Caillot D, Casasnovas RO, Douvier S, Nivelon-Chevallier A: Exposure to hydroxyurea during pregnancy: a case series. Leukemia 2001;15:1309-1311.

23 Ring AE, Smith IE, Jones A, Shannon C, Galani E, Ellis PA: Chemotherapy for breast cancer during pregnancy: an 18-year experience from five London teaching hospitals. J Clin Oncol 2005;23:4192-4197.

24 Basaran D, Turgal M, Beksac K, Ozyuncu O, Aran O, Beksac MS: Pregnancy-associated breast cancer: clinicopathological characteristics of 20 cases with a focus on identifiable causes of diagnostic delay. Breast Care 2014;9:355-359.

25 Amant F, Deckers S, Van Calsteren K, Loibl S, Halaska M, Brepoels L, Beijnen J, Cardoso F, Gentilini O, Lagae L, Mir O, Neven P, Ottevanger N, Pans S, Peccatori F, Rouzier R, Senn HJ, Struikmans H, Christiaens MR, Cameron D, Du Bois A: Breast cancer in pregnancy: recommendations of an international consensus meeting. Eur J Cancer 2010;46:3158-3168.

26 Gwyn KM, Theriault RL: Breast cancer during pregnancy. Curr Treat Options Oncol 2000;1:239-243.

27 Berry DL, Theriault RL, Holmes FA, Parisi VM, Booser DJ, Singletary SE, Buzdar AU, Hortobagyi GN: Management of breast cancer during pregnancy using a standardized protocol. J Clin Oncol 1999;17:855-861.

28 Cheng YK, Zhang F, Tang LL, Chen L, Zhou GQ, Zeng MS, Kang TB, Jia WH, Shao JY, Mai HQ, Guo Y, Ma J: Pregnancy associated nasopharyngeal carcinoma: a retrospective case-control analysis of maternal survival outcomes. Radiother Oncol 2015;116:125-130.

29 Lin TI, Lin JC, Ho ES, Chou MM: Nasopharyngeal carcinoma during pregnancy. Taiwan J Obstet Gynecol 2007;46:423-426.

30 Ganapathi KA, Paczos T, George MD, Goodloe S, Balos LL, Chen F: Incidental finding of placental choriocarcinoma after an uncomplicated term pregnancy: a case report with review of the literature. Int J Gynecol Pathol 2010;29:476-478.

31 Ghaemmaghami F, Karimi Zarchi M: Early onset of metastatic gestational trophoblastic disease after fullterm pregnancy. Int J Biomed Sci 2008;4:74-77.

32 Bratila E, Ionescu CA, Vladescu CT, Cirstoiu MM, Berceanu C: Gestational choriocarcinoma after term pregnancy: a case report. Rom J Morphol Embryol 2015;56:267-271. 